\title{
Urgence et mélancolie dans le travail social
}

Nicolas Amadio

\section{OpenEdition}

Journals

Édition électronique

URL : http://journals.openedition.org/communicationorganisation/3412

DOI : 10.4000/communicationorganisation.3412

ISSN : $1775-3546$

\section{Éditeur}

Presses universitaires de Bordeaux

\section{Édition imprimée}

Date de publication : 1 juin 2006

Pagination : 206-219

ISSN : 1168-5549

\section{Référence électronique}

Nicolas Amadio, «Urgence et mélancolie dans le travail social », Communication et organisation [En

ligne], 29 | 2006, mis en ligne le 21 juin 2012, consulté le 18 décembre 2020. URL : http://

journals.openedition.org/communicationorganisation/3412 ; DOI : https://doi.org/10.4000/

communicationorganisation.3412

Ce document a été généré automatiquement le 18 décembre 2020.

(C) Presses universitaires de Bordeaux 


\title{
Urgence et mélancolie dans le travail social
}

\author{
Nicolas Amadio
}

1 À quelles conditions et à quel prix une activité professionnelle fondée sur la notion de service est-elle possible dans l'urgence? En nous appuyant sur plus d'une soixantaine d'entretiens semi-directifs effectués auprès de travailleurs sociaux employés par une institution territoriale, nous nous attacherons à mettre en perspective le lien existant entre le travail dans l'urgence et l'apparition de la mélancolie ${ }^{1}$ comme expression de la démotivation. En effet, les spécificités liées à la pratique professionnelle du travail social dans ce type d'organisation ont, grâce à une analyse socio-psychologique (De Gaulejac, 1993) attentive aux phénomènes émotionnels (Travailler, 2002), permis de relever différents processus et stratégies d'adaptation face à l'injonction d'un travail dans l'urgence.

2 En redéfinissant l'impression vague de "malaise » à partir des phénomènes émotionnels que sont l'angoisse, la peur et la crainte, on comprendra d'une part l'ambiguïté de la position des travailleurs sociaux dans cette organisation et d'autre part les difficultés de communication qui caractérisent leurs relations. Dans ce contexte, la notion d'urgence intervient, du point de vue des travailleurs sociaux, comme un élément aussi bien interne qu'externe à l'organisation menaçant leur autonomie et la dimension non-objective de leur pratique. En tant qu'élément externe, elle accroît un sentiment d'insatisfaction que traduit une tristesse largement éprouvée. En tant qu'élément interne, multipliant les procédures d'urgence, l'organisation administrative impose aux travailleurs sociaux de rendre compte de manière objective de leur efficacité. Cette injonction les renvoie alors directement à la double dimension du sentiment d'infinitude de la pratique du travail social : infinitude en termes de sens, infinitude en termes de temps. Confrontés à l'impossibilité de communiquer avec leur organisation, focalisés sur la relation d'aide et l'investissement affectif qui leur est inhérent, les travailleurs sociaux expriment des formes de démotivation qui rappellent la dimension mélancolique des pathologies dépressives. De plus en plus courantes au sein des métiers de service, ces formes dépressives des psychopathologies au travail 
(Desjours, 1991) trouvent ainsi en partie leur ressort dans l'imposition de processus organisationnels caractérisés par l'urgence.

\section{Malaise dans le travail social ?}

3 Afin d'appréhender les rapports qu'entretiennent urgence et mélancolie dans le travail social, il nous faut commencer par présenter le contexte dans lequel ils s'inscrivent: celui d'une organisation particulière, d'un service social d'une collectivité territoriale. Notre fréquentation du terrain a en effet fait émerger une question qui dessinera la perspective que nous emprunterons pour ce faire: ce que les travailleurs sociaux ressentent au sein de l'organisation correspond-il au sentiment de malaise que nombre d'ouvrages spécialisés en matière de travail social (Ion, Tricat, 1998) dénoncent ? A la lumière des manifestations émotionnelles que nous avons pu observer ${ }^{2}$, le malaise nous semble pouvoir se définir comme l'enchevêtrement de trois phénomènes émotionnels entretenant des relations étroites : l'angoisse, la peur et la crainte.

\section{L'angoisse, la peur et la crainte à la source du sentiment de malaise}

4 La non-lisibilité de la position des travailleurs sociaux au sein de la collectivité territoriale fait ressentir une forme d'angoisse certes latente mais qu'il est possible de saisir lors de l'apparition de conflits résultant d'une imposition quelconque émanant de la hiérarchie. La puissance et le pouvoir dont semble disposer cette hiérarchie et le caractère indiscutable de ses décisions, associés à l'impression qu'elle est soutenue par une réglementation à toute épreuve, fait émerger chez les travailleurs sociaux une angoisse de persécution pouvant se traduire en une forme dépressive (démotivation).

Daniel Anzieu (1971) remarquait à juste titre qu'une des fonctions de la réglementation dans les organisations était de garantir la défense des individus contre ces angoisses. Or, les travailleurs sociaux, du fait de leur incapacité à se situer au sein du système administratif, ne se sentent pas protégés au même titre que leurs supérieurs par la réglementation. Dès lors, l'angoisse peut se développer et tout mouvement vertical se dirigeant du haut vers le bas du système hiérarchique pyramidal est vécu comme un signe de menace. La conjonction d'une large autonomie dans l'exercice quotidien du travail et de la rigidité d'un cadre administratif dans lequel il vient s'inscrire peut ainsi donner lieu à une angoisse s'exprimant de manière individuelle car c'est à ce niveau que se ressent le plus l'autonomie et ce, notamment, par rapport à la relation d'aide, pratique professionnelle qui fait la spécificité du travail social. Le flou entourant les pratiques professionnelles des travailleurs sociaux favorise ainsi l'apparition d'une forme d'angoisse qui sera d'autant plus vive que le travailleur social se sentira éloigné du système administratif. L'opposition entre un système administratif rigide et une pratique professionnelle faisant particulièrement preuve d'autonomie permet difficilement aux travailleurs sociaux de s'adapter au cadre de leur fonction (bien qu'ils le soient à leur tâche).

Mais le malaise que ressentent les travailleurs sociaux ne s'exprime pas uniquement à travers cette angoisse. Ces derniers font par ailleurs montre d'une peur renvoyant à l'indicible et au fantasme d'une administration toute puissante. Ainsi les travailleurs 
sociaux sont-ils soumis à la peur d'un système dans lequel ils se sentent entraînés un peu malgré eux, poussés par des forces, des puissances et surtout un pouvoir qu'ils ne parviennent pas à déterminer et dont les formes concrètes restent indéfinies. Cette peur vient se conjuguer à l'angoisse en même temps qu'elle en diffère de par son caractère soudain, éphémère. Elle semble résulter de la prise de conscience et de la transposition d'une situation fantasmée, celle d'un pion pris dans les fers d'une toute puissance, dans la réalité. Si l'angoisse résulte en premier lieu de l'impression de ne pas être protégé par le règlement du système administratif, la peur qu'expriment les travailleurs sociaux provient avant tout de leur méconnaissance de ce système, en ce qu'elle permet l'élaboration fantasmatique d'une puissance et d'un pouvoir extraordinaires. L'impersonnalité et la froideur des relations que supposent les travailleurs sociaux au sein de l'administration favorisent par ailleurs cette impression d'indicibilité, d'impossibilité de repérer et d'identifier quelqu'un comme auteur ou responsable ${ }^{3}$.

7 Ainsi l'angoisse résulterait du fait de se questionner à propos de ses propres possibilités de défense et de protection, de se reposer sur le règlement, tandis que la peur découlerait plutôt de la réification de la puissance offensive. Une telle émotion ne connaît bien sûr pas la permanence et la latence avec laquelle se manifeste l'angoisse. Elle reste exceptionnelle, mais il nous semble que les quelques manifestations que nous avons pu déceler montrent bien à quel point les acteurs de l'organisation peuvent être amenés à souffrir de leur position.

8 La hiérarchie et l'impression de se confronter à une «machine énorme » sont la source essentielle d'une crainte qui s'exprime à travers la peur d'être sanctionné pour un comportement non adéquat, un manquement ou une faute professionnelle. L'autorité administrative, hiérarchique, repose essentiellement sur l'effet d'imposition et de lourdeur de la réglementation. N'ayant que très peu de connaissances concernant le fonctionnement du système administratif et étant confronté à l'impersonnalité des relations, ce dont les travailleurs sociaux n'ont guère l'habitude puisqu'ils privilégient les rapports frontaux -allant même jusqu'à en faire une spécialité (cf. la relation d'aide)- ils n'ont d'autres possibilités que d'éprouver la crainte de mal faire.

9 L'exigence de l'administration, au-delà de la frustration, suscite un sentiment de crainte qui se vérifie par le recours fréquent aux conseils de leur supérieur hiérarchique direct ${ }^{4}$. Cette crainte vient encore une fois souligner la position inconfortable des travailleurs sociaux et l'attention qu'ils y portent dans la mesure où elle est le signe non pas d'un détachement total vis-à-vis de l'administration, mais d'une volonté d'être bien vu et de ne pas décevoir ses supérieurs. La crainte d'avoir mal fait, de recevoir des sanctions, nous informe par ailleurs sur le sentiment de compétence des travailleurs sociaux par rapport aux exigences du système administratif. Partant, nous pouvons émettre l'hypothèse selon laquelle les travailleurs sociaux, du fait de leur manque de connaissance du système administratif, estiment ne pas avoir de réelles compétences en la matière, ce qui contribue à les rendre plus vulnérables aux menaces que fait peser sur eux ce dernier.

L'angoisse, la crainte et la peur sont des émotions inextricablement liées mais qui permettent, en cherchant à en définir les causes et à les articuler entre elles ainsi qu'à d'autres phénomènes organisationnels, de comprendre comment un sentiment de malaise a pu se développer dans l'organisation. Elles se rapportent à une dimension organisationnelle principalement structurée par les contradictions résultant de 
l'opposition entre un monde de l'administration et un monde du travail social, alors que la tristesse et la mélancolie renvoient respectivement aux rapports de ce dernier avec des éléments soit externes soit internes à l'organisation parmi lesquels l'urgence se manifeste par ses différentes conséquences.

\section{La tristesse comme forme d'expression de l'insatisfaction au travail.}

11 Remettant en question les fondements professionnels de l'approche de terrain, la tristesse est mise à l'écart par les travailleurs sociaux qui entretiennent à son égard une attitude ambiguë. Ainsi, si d'un côté la tristesse éprouvée par la connaissance des situations difficiles que vivent les usagers et par la misère sociale en général apparaît comme un moteur et même une raison d'agir et de s'engager en leur faveur, de l'autre, le travailleur social ne peut se défendre d'être affecté par cette dernière même si, à force d'y être confronté et de la côtoyer, certains ont su établir des procédures cognitives leur permettant de la mettre à l'écart.

12 La présence de cette émotion tient non seulement au fait qu'il y ait constatation de la misère sociale, mais aussi au fait que le travailleur social s'engage, parallèlement, dans un processus d'identification. L'investissement personnel du travailleur social sera en effet d'autant plus fort, non pas forcément que la misère de la situation qu'il constate l'est aussi, mais qu'il n'a d'autres moyens d'y répondre qu'à travers sa propre personne.

13 La tristesse devient une émotion contagieuse lorsque le processus d'évaluationidentification qui en est producteur est sollicité pour instaurer une dynamique d'intervention, d'engagement de soi. En d'autres termes, si une émotion comme la tristesse est un mouvement de l'âme, qu'elle traduit le fait de se jeter hors de soi, il est des conditions dans lesquelles elle exprime la volonté de se jeter en l'autre. Partant, le refoulement de la tristesse donnera parallèlement lieu à une série d'interrogations en termes d'éthique, d'objectivité, de neutralité et de professionnalisme dans la pratique et l'intervention du travail social ; notamment lorsqu'elles sont mises en rapport avec l'engagement militant des travailleurs sociaux et avec leur aptitude à se mettre aux cotés des usagers et à défendre leurs droits. Pensant parvenir à objectiver de cette manière une émotion dont la présence est quasi permanente, les travailleurs sociaux voient dans le terme même d'éthique un moyen d'échapper aux questions personnelles et individuelles qu'elle soulève.

Ramenant le débat de l'identification du travailleur social à l'usager dans une dimension collective, les travailleurs sociaux parviennent à éviter une émotion qui n'est pas non plus sans renvoyer à une forme d'insatisfaction par rapport au travail qui est accompli. La tristesse, subie et éprouvée avec une certaine continuité par les travailleurs sociaux de terrain, peut donc faire l'objet de stratégies de contournement, mais ces derniers ne peuvent agir de façon à l'éradiquer. Cette incapacité est non seulement liée au fait qu'elle se comprend essentiellement dans une dimension individuelle, personnelle, mais aussi en ce qu'elle provient non pas de l'intérieur de l'organisation mais de l'extérieur de cette dernière.

15 L'extériorité des stimuli provoquant la tristesse entraîne l'impossibilité des travailleurs sociaux à agir directement sur elle et l'obligation de développer des stratégies personnelles pour y faire face. On peut ainsi émettre l'hypothèse que cette émotion 
exprime une insatisfaction des travailleurs sociaux de terrain renvoyant directement à des éléments extérieurs à l'organisation, à leur confrontation à la misère et la pauvreté sociale, ainsi qu'à leur engagement personnel dans leur pratique professionnelle (Mias, 1998).

Parmi ces éléments extérieurs, l'urgence avec laquelle les usagers présentent leurs demandes accroît cette insatisfaction dans la mesure où l'une des principales sources de cette dernière provient justement de leur incapacité non pas à faire face mais à répondre avec des moyens appropriés, définis et réglementés, avec des outils spécialisés à ces situations sociales. En effet, après avoir épuisé toutes les demandes, ou simplement constaté le désarroi d'un usager dont la situation ne correspond pas aux critères de catégorisation définis par l'organisation, le travailleur social n'a plus comme recourt que sa propre personne pour investir la relation d'aide. Sans mettre en doute la souffrance des usagers, les travailleurs sociaux constatent avec une certaine amertume que l'urgence est plus instrumentalisée par ces derniers que réellement subie. Si elle semble répondre à un usage de l'institution devenant de plus en plus fréquent et en partie provoqué par elle (nous reviendrons sur ce point qui renvoie à la dimension interne de l'organisation), il reste que, lorsque les travailleurs sociaux sont directement confrontés à ces urgences, c'est à travers un fort investissement personnel qu'ils tentent d'y répondre. Or, le manque de ressources, en termes de moyens et de procédures, pour répondre à la misère sociale est une première source d'insatisfaction des travailleurs sociaux qui voient ainsi leur tristesse s'accroître par la perspective de la non-résolution des problèmes sociaux de l'usager et donc celle de leur aggravation. Le caractère urgent des situations ne fait alors qu'amplifier l'impression d'accélération de cette aggravation.

Ne restant plus au travailleur social que sa propre personne à mettre en médiation dans la relation d'aide qu'il tient malgré tout à entretenir et à faire fructifier, il a cependant pleinement conscience du peu de succès d'un tel investissement de soi. Si l'on peut penser que c'est le fait d'éprouver dans un premier temps de la tristesse par rapport à la misère sociale constatée qui a poussé le travailleur social à s'engager et à agir, il nous semble que c'est à présent à une forme de culpabilité qu'il doit son obstination et l'implication de sa propre personne. Soulignons par ailleurs que, plus il s'engage personnellement dans la relation d'aide, plus sa culpabilité de ne pouvoir répondre aux attentes de l'usager va être grande. Le sentiment de devoir agir dans l'urgence contraint alors encore un peu plus les travailleurs sociaux à s'impliquer personnellement. Par conséquent, un échec dans ce cadre acquiert une intensité dramatique d'autant plus prégnante et aboutit, finalement, à un sentiment d'insatisfaction renvoyant à une incapacité personnelle de pouvoir résoudre les problèmes qui y sont soulevés. En ce sens, les travailleurs sociaux se sentent tristes, tristes parce qu'insatisfaits de ne pouvoir répondre, de se sentir dépassés par le caractère urgent que prennent les situations. Sur un plan collectif, cette tristesse est traduite par un certain nombre d'interrogations sur la légitimité du travail social (Hatzfeld, 1998), qui orientent le débat vers des éléments internes à l'organisation.

18 L'investissement de leur personne les entraîne, au-delà d'une simple implication professionnelle dans l'intervention sociale, dans un processus d'individuation, de personnalisation de la pratique professionnelle, qui est, en outre, favorisé par le manque d'outils et de moyens d'intervention propres à jouer le rôle de médiateur dans la relation de face à face, mais aussi par le caractère urgent des situations présentées 
par les usagers. La tristesse qu'ils éprouvent est ainsi une émotion à la fois personnelle et sociale, car relative à la représentation que se fait le travailleur social de sa place ou de son utilité dans la société et sur laquelle des éléments extérieurs à l'organisation ont une importante influence. Là où, pour les usagers, l'urgence fait fracas, elle se fait chaque jour plus insidieuse pour les travailleurs sociaux.

\section{La mélancolie comme forme d'expression de la démotivation au travail.}

19 Alors que le sentiment de malaise, que nous avons essayé de comprendre comme amalgame d'émotions fondamentales que sont l'angoisse, la peur et la crainte, nous a semblé se rapporter largement à l'organisation dans son acception en termes d'opposition et / ou d'imbrication du travail social dans le cadre administratif, la tristesse nous a paru faire principalement référence à des éléments provenant de l'extérieur de l'organisation. Aussi pensons-nous que, dans notre effort de redéfinition générale et de réinterprétation du sentiment de malaise, il importe de chercher à mettre en perspective quelle émotion pourrait être finalement exprimée à travers des phénomènes organisationnels ressentis par les travailleurs sociaux qui sont à la fois relativement proches de ce que nous avons entendu sous le terme de tristesse et significativement divergents de cette dernière en ce qu'ils trouvent leur source à l'intérieur de l'organisation. Conforté par le fait que nous avions là affaire à plusieurs signes de démotivation plus que d'insatisfaction, nous pensons que l'on peut suggérer d'interpréter ces impressions, ces ressentis, ces émotions éprouvées, comme un ensemble d'éléments exprimant une certaine mélancolie ${ }^{5}$.

\section{L'infinitude du travail social en termes de temps et de sens.}

Avant d'en arriver à n'avoir comme dernier recourt que leur propre personne, les travailleurs sociaux disposent tout de même, et de plus en plus, de moyens d'aide et d'intervention prenant pour la plupart la forme de procédures administratives. Le nombre et la variété de ces procédures ne dispensent pas d'une certaine monotonie dans leur mise en application. Le travailleur social se voit ainsi contraint, et s'en plaint, de devoir remplir de nombreux formulaires administratifs en de multiples exemplaires tandis qu'il a à faire face aux enjeux toujours singuliers de la situation sociale d'un individu ou d'une famille en particulier. Face à cette contradiction à laquelle il n'a guère de moyen d'échapper autrement que par la possibilité de multiplier les suivis et les accompagnements lui donnant l'occasion de restituer à l'usager sa personnalité, une impression de démotivation commence à poindre. L'intrusion du cadre administratif dans la relation d'aide tend à dépersonnaliser l'usager et le fait de rédiger avec une certaine répétitivité les mêmes formulaires amène certains travailleurs sociaux à se sentir envahis par l'administratif, situation contre laquelle ils répondent énergiquement en brandissant non seulement les vertus et l'intérêt de la relation d'aide, mais aussi son caractère traditionnel dans la pratique de l'intervention sociale, c'est-à-dire sa légitimité en tant que savoir-faire, en tant que « spécialité ». 
21 Cette impression d'envahissement sera vécue comme une attaque de la part de l'administration et de la hiérarchie en général contre des pratiques professionnelles dans lesquelles ils trouvent le plus d'autonomie et qui va jusqu'à représenter, comme le souligne François Dubet, « la référence ultime, c'est la relation, toujours perçue comme le sel du métier, comme son espace héroïque, comme celui qui signe une spécificité professionnelle» (Dubet, 2002: 262). Pourtant, si cette colonisation de l'acte administratif donne de temps à autre lieu à de vives émotions comme la colère dans le cas de situations où elle peut être particulièrement visible et frustrante, son intégration dans le quotidien des travailleurs sociaux, mêlée à la peur qu'ils ressentent de leur hiérarchie indirecte et à l'angoisse suscitée par l'indiscernabilité des limites et de leur propre position, les pousse à accepter cet état de fait. L'ultime référence, comme l'appelle François Dubet, que constitue la relation d'aide reste pour bien des travailleurs sociaux leur espace de liberté dans l'organisation, le lieu et le temps dans lequel ils ont la possibilité d'exercer leur activité professionnelle en dehors de la contrainte et de la monotonie administrative, là où l'organisation, l'équipe, la hiérarchie, l'administration s'éloignent pour laisser place à la satisfaction, au désir et à la motivation de travailler sur et avec l'autre. Ainsi, sa mise à l'écart progressive par l'administration qui encourage un traitement dans l'urgence des demandes d'aide sociale à travers leur prise en charge non plus par les travailleurs sociaux mais par des formulaires administratifs, favorise une forme de dysrythmie dont l'expression la plus manifeste réside en un enlisement dans le passé.

22 Ce passé, magnifié par l'importance qu'y aurait eu la relation d'aide, montre bien que c'est autour de cette dernière que se dessine une rythmique qui soutient la pratique professionnelle des travailleurs sociaux. Le déséquilibre de cette rythmique, parce qu'il consiste en une attaque de la relation d'aide, de son noyau porteur, entraîne les travailleurs sociaux à avoir le sentiment de ne pas pouvoir suivre les affaires en cours, ni le rythme des activités quotidiennes. Ils éprouvent alors un sentiment de perte (Aubert, 2004) de contrôle du temps et finalement de soi, dans la mesure où c'est autour de la relation d'aide que les travailleurs sociaux se représentent leur pratique.

Cependant, l'expérience qu'ils peuvent faire de tels sentiments ne s'arrête pas à ce phénomène de dysrythmie. Agissant dans une organisation qu'ils disent n'avoir pas choisi mais envers laquelle ils reconnaissent tout de même ne serait-ce que le fait qu'elle leur apporte une certaine garantie d'emploi ainsi qu'une autonomie certaine, les travailleurs sociaux ne parviennent pas eux-mêmes à saisir réellement l'efficacité ou l'utilité de leur pratique professionnelle. Ils attendent pourtant du système administratif une certaine forme de reconnaissance ou, au moins, qu'on leur permette d'identifier ce qu'ils auraient contribué à produire. Le caractère indéfini du travail social amène les travailleurs sociaux non pas à se sentir inutiles puisqu'ils trouvent quelques motivations dans la relation d'aide, mais enclins à épouser une certaine forme de démotivation.

La mélancolie qu'éprouvent les travailleurs sociaux semble donc avoir pour entre autre cause la non reconnaissance de l'utilité de leur travail. Le décalage existant entre l'urgence dans laquelle ils se voient contraints de travailler par le système administratif et le peu de signes objectifs qui leur est rendu d'avoir produit quelque chose augmente considérablement l'impression qu'ils peuvent se faire de la durée de leur pratique. En effet, le fait qu'il existe peu de moments pendant lesquels les travailleurs sociaux parviennent à constater objectivement, comme une donnée en soi, le résultat positif de 
leur travail, tandis qu'ils sont de plus en plus amenés à travailler dans l'urgence et à répondre rapidement aux demandes d'aides des usagers, contribue à faire diminuer la valeur de l'unité de temps et, par conséquent, à augmenter le caractère peu productif du travail social, ce qui n'est pas sans favoriser le ressentiment d'une certaine mélancolie (Ion, 1998). Sentiment de monotonie, impression de ne plus être en mesure de jouir de sa pratique professionnelle, de s'associer aux émotions des autres et du monde sont autant de signes de la dimension mélancolique.

\section{Rapidité des changements et urgence dans le travail social : quand l'avenir se fait angoissant.}

Le travail social connaît des changements et des remises en question profondes tant aux niveaux technique qu'organisationnel. La mise en place de procédures administratives semblant répondre à de nouvelles thématiques ayant cours dans les politiques publiques telles qu'elles peuvent s'entendre sous les termes de projet ou de contrat, favorise par ailleurs une pratique professionnelle fondée sur le travail dans l'urgence. En d'autres termes, les travailleurs sociaux ont le sentiment d'être plus que contraints, impuissants, face aux mutations de leur pratique professionnelle.

Le peu de poids qu'ils ont non seulement dans l'élaboration (qui dépend essentiellement des élus politiques) mais aussi dans l'application (qui dépend essentiellement des administrateurs) des nouvelles politiques publiques en matière d'intervention sociale et qui viennent marquer significativement leurs pratiques quotidiennes leur donne un amer sentiment de démotivation. Ainsi, la démotivation que peuvent éprouver certains travailleurs sociaux provient-elle en partie aussi du fait qu'il leur est donné de ressentir de façon quotidienne les efforts que met en œuvre l'organisation pour les encadrer, faisant en sorte de limiter leur possibilité d'action réelle et altérant le sens de leur travail (Hanique, 2004).

Les mutations du travail social sont plus subies qu'agies par les travailleurs sociaux et, de facto, ne parvenant pas à s'inscrire dans ces dernières, ils sont d'autant plus enclins à éprouver leur inadaptation à l'organisation ou, du moins, à son fonctionnement. On comprend alors aisément qu'ils puissent perdre toute motivation personnelle et professionnelle pour leur travail. L'institution, en faisant de l'urgence le noyau central autour duquel vont s'organiser les pratiques professionnelles des travailleurs sociaux, organise en fait autour d'eux un faisceau d'éléments concourant à briser un équilibre bien fragile. Croyant pouvoir améliorer l'efficacité du travail social sans en connaître les spécificités, elle instrumentalise l'urgence pour construire autour d'elle un ensemble de procédures administratives loin de satisfaire non seulement à l'ambition des travailleurs sociaux mais plus encore à leur identité fondamentale.

De plus, la rapidité avec laquelle se cumulent ces transformations renforce le sentiment de perte de contrôle et de dépossession que manifestent les travailleurs sociaux. Loin d'être auteurs et alors qu'ils parvenaient à jongler entre le fait d'être acteurs à la fois dans et de l'institution, cette dernière, en utilisant la pression qui constitue l'urgence, les contraint à se faire agents. Se mettent alors en exergue des difficultés à se mettre au travail, à établir des projets, à penser l'avenir d'une manière positive ${ }^{6}$. Le futur devient une menace, il n'est plus abordé sous le sceau de l'ambition et de la dynamique, mais fait retour sur une angoisse que nous avons déjà pu aborder ci-dessus. C'est par la 
conjugaison de l'ensemble de ces éléments que les travailleurs sociaux font l'expérience de la mélancolie.

Finalement, l'espoir et l'intérêt qu'ils accordent aux mutations de leurs pratiques professionnelles relèvent plus de la rêverie accompagnant l'état d'abattement et de vague tristesse qui définissent la mélancolie ${ }^{7}$ que d'un réel engouement. Les travailleurs sociaux sont ainsi dans une sorte de présence-absence vis-à-vis de l'organisation. S'ils épousent ses formes, s'ils semblent s'adapter aux modèles qui leurs sont proposés, ce n'est pas tant grâce au génie gestionnaire du système administratif que par la pseudo-résignation qu'exprime leur mélancolie. Aussi convient-il de ne pas confondre sur ce point ce qui tient du pouvoir de l'organisation comme déterminant les comportements et les actions des travailleurs sociaux de ce qu'il est tenu de rapporter à l'autonomie de ces derniers. En effet, nous n'avons eu de cesse de rappeler l'ambivalence de ce sentiment qui permet d'exprimer à la fois des formes de démotivation issues des contraintes et des déterminations d'action que fait peser l'organisation sur les acteurs, en même temps qu'un souhait d'agir ou de maintenir son engagement, non pas envers cette dernière, mais envers une pratique professionnelle qui s'inscrit dans son cadre.

\section{Conclusion}

Notre brève analyse de la mélancolie éprouvée par les travailleurs sociaux nous aura permis de mettre en perspective plusieurs processus organisationnels concourant à sa production. Parmi eux, l'urgence intervient à la fois comme élément interne et externe à l'organisation en accélérant ce phénomène de mélancolisation. Mis en rapport avec les éléments internes à l'organisation et plus généralement avec le cadre administratif, le phénomène émotionnel de mélancolie dont nous avons cherché les causes et les effets aussi bien au niveau de l'organisation que de l'acteur qui l'éprouve, offre la possibilité de comprendre la place du travailleur social au sein de l'organisation à travers la présence de différents processus. Nous aurons pu observer que l'urgence accompagne et intensifie les effets : de la répétitivité des actes administratifs vis-à-vis de la relation singulière d'aide, de l'incapacité des travailleurs sociaux à reconnaître et faire reconnaître par le système administratif leur utilité, de leur impuissance face aux mutations du travail social que se charge de mettre en place le système administratif ou encore de leur impression de morcellement en catégories d'ordre administratif. Autant de processus qui ont pu révéler en partie, les multiples raisons d'une démotivation des travailleurs sociaux envers leurs pratiques professionnelles. Cette démotivation, que nous renvoyons au phénomène émotionnel de mélancolie dont l'essence nous a paru être une présence-absence caractérisant la focalisation de soi en tant que volonté d'engagement sur un élément précis (ici la relation d'aide) autour duquel les choses n'observent alors d'autres importances que leur gravitation, se distingue ainsi non seulement du phénomène émotionnel de tristesse que de celui du sentiment de malaise renvoyant à l'articulation de l'angoisse, de la peur et de la crainte. 


\section{BIBLIOGRAPHIE}

Anzieu D., 1971, Le groupe et l'inconscient. L'imaginaire groupal, Paris, Dunod.

Aubert N., 2004, Le culte de l'urgence, la société malade du temps, Paris, Flammarion.

Castro D. (dir.), 2004, Les interventions psychologiques dans les organisations, Paris, Dunod.

Dubet F., 2002, Le déclin de l'institution, Paris, Seuil, p. 262.

Garbarini J., 1997, Relation d'aide et travail social, ESF, Paris.

Gaulejac V. (de), 1993, Sociologie Cliniques, Paris, Hommes et Perspectives.

Hanique F., 2004, Le sens du travail, Chronique de la modernisation au guichet, coll. « sociologie

clinique ", Paris, Erès.

Hatzfeld H., 1998, Construire de nouvelles légitimités en travail social, Paris, Dunod.

Ion J., 1998, Le travail social au singulier, Paris, Dunod.

Ion J., Tricat J.-P., 1998, Les travailleurs sociaux, Paris, Repères, La Découverte.

Laïdi Z., 1998, « L'urgence comme dévalorisation culturelle de l'avenir », dans Soulet M.-H. (dir.), Urgence, Souffrance, misère, Lutte humanitaire ou politique sociale, EUFS, Suisse.

Mias C., 1998, L'implication professionnelle dans le travail social, Paris, L'Harmattan.

Sociétés, 2004, Mélancolie Sociale, n 86, Bruxelles, De Boeck.

Travailler, Revue Internationale de Psychopathologie et de Psychodynamique du Travail, 2002, Les

émotions dans le travail, $n^{\circ}$ 9, Paris, Martin Média.

\section{NOTES}

1. Notre acception de la notion de mélancolie renvoie à une lecture sociologique en termes de phénomène émotionnel que nous ne détaillerons pas ici. Cependant, sa mise en rapport à la démotivation et à différents facteurs ou processus propres au «terrain » en question donne un aperçu de la perspective dans laquelle nous l'employons.

2. Pour un aperçu bref et convaincant de « la place des émotions en milieu professionnel » voir le chapitre du même titre de Castro (2004 : 91-108).

3. Joëlle Garbarini montre bien que les travailleurs sociaux voient « l'aide administrative comme une aide ponctuelle, comme une relation à éviter, car le risque est de devenir inhumaine, bureaucrate, insensible aux autres. L'image même de l'administration les inquiète, leur fait peur. » Garbarini (1997 : 48).

4. Dénommés "Responsable de Circonscription ", ils sont le plus souvent eux-mêmes issus du travail social de terrain.

5. «La mélancolie, concept princeps désignant un état dépressif particulièrement sévère, caractérisé par une douleur morale intense, accompagnée de sentiments très prononcés de culpabilité, d'auto-dépréciation et de tristesse.» Aubert (2004: 158). Pour une approche en termes de "mélancolie sociale" voir le numéro de la revue Société (2004).

6. Voir l'article « L'urgence ou la dévalorisation culturelle de l'avenir » de Laïdi Z. (1998 : 43-61) 
7. Ainsi trouve-t-on dans Le Petit Robert, édition de 1995, la mélancolie définie comme un « état d'abattement, de tristesse vague, accompagnée de rêverie » ou encore comme la «bile noire, l'une des quatre humeurs, dont l'excès, selon la médecine ancienne, poussait à la tristesse ».

\section{RÉSUMÉS}

Cet article a pour ambition de mettre en exergue les relations que peuvent entretenir deux phénomènes comme l'urgence et la mélancolie dans un contexte organisationnel particulier, celui du service social d'une institution territoriale. Dans la perspective d'une sociologie des phénomènes émotionnels, nous analyserons différents processus organisationnels liés aux spécificités du travail social. L'urgence apparait alors comme un facteur aggravant des phénomènes émotionnels comme la tristesse et la mélancolie qui sont l'expression d'éléments d'insatisfactions et de démotivations renvoyant à un sentiment de malaise qui a pu se développer dans l'organisation.

The aim of this paper is to expose two particular phenomena such as urgency and melancholy and to see the kind of relations they maintain in the particular context of a territorial social service. Our framework (i.e. sociology of emotions) will take into account different organisational processes wich one are directly linked to the specificities of social work. Within this perspective, urgency appears to be an aggravating factor of emotional phenomena such as sadness and melancholy. These phenomena are the expression of dissatisfaction and lack of motivation and are in strict relationship with the discontent that could have been developped in the organisation.

\section{INDEX}

Mots-clés : mélancolie, organisation, travail social

\section{AUTEUR}

\section{NICOLAS AMADIO}

Nicolas Amadio est Allocataire-Moniteur de Recherche à l'Université March Bloch de Strasbourg. Son domaine de recherche est la sociologie des émotions, du travail et des organisations. Mail : Nicolas.Amadio@umb.u-strasbg.fr 